\title{
A simulation case study to improve staffing decisions at mass immunization clinics for pandemic influenza
}

\author{
Michael F Beeler*, Dionne M Aleman and Michael W Carter \\ University of Toronto, Toronto, ON, Canada
}

\begin{abstract}
Mass immunization clinics (MICs) are an important component of pandemic influenza control strategies in many jurisdictions. Decisions about staffing levels at MICs affect several factors of concern to public health authorities: total vaccination volume, patient wait-times, operating costs, and intra-facility influenza transmission risk. We present a discrete-event simulation of an MIC to assess how strongly staffing changes affect these factors. The simulation is based on data from Canadian clinics responding to pandemic H1N1 in 2009. This study is the first to model flu transmission risk at an MIC, and the first to relate such risk to staffing decisions. We show that the marginal benefit of adding staff is greatly underestimated if indirect waiting costs and intra-facility infections are not considered.
\end{abstract}

Journal of the Operational Research Society (2014) 65, 497-511. doi:10.1057/jors.2013.148

Published online 27 November 2013

Keywords: simulation; health service; mass vaccination; pandemic influenza; infection control

\section{Introduction}

Vaccination is an important component of pandemic influenza plans in many jurisdictions (Centers for Disease Control and Prevention, 2011; Public Health Agency of Canada, 2011; United Kingdom Department of Health, 2011). During the $2009 \mathrm{H} 1 \mathrm{~N} 1$ pandemic, mass immunization clinics allowed for the rapid, large-scale administration of vaccines without overwhelming hospitals, regular walk-in clinics, and physician offices. Since vaccine development and distribution can take between 4 and 6 months from the onset of the pandemic (United Kingdom Department of Health, 2011), vaccines may become available only in the pandemic's later stages, as was the case with the $2009 \mathrm{H} 1 \mathrm{~N} 1$ vaccine, which started being administered in North America a couple weeks before the peak of the pandemic's second wave in November 2009.

The delayed availability of the vaccine has two important consequences. First, the effectiveness of the vaccination programme depends not only on the total percentage of the population vaccinated, but also on the programme's speed, as vaccinations given later in the pandemic have less value than those delivered earlier (Mylius et al, 2008). High-volume mass immunization clinics (MICs) offer such speed, but require high levels of staffing to meet public demand. Second, the vaccination programme will take place in the context of a healthcare system burdened by influenza cases. Pandemics increase demand for healthcare workers ( $\mathrm{HCWs}$ ), and may also decrease supply due to flu cases among HCWs (Mitchell et al, 2012).

*Correspondence: Michael F Beeler, Department of Mechanical and Industrial Engineering, University of Toronto, 5 King's College Road, Toronto, ON, M5S $3 G 8$ Canada.
Consequently, public health authorities must make difficult decisions about how many staff to allocate to MICs, possibly at the expense of other areas of the healthcare system.

Through discussions with Niagara Region Public Health (NRPH) and Toronto Public Health, two public health units in Ontario, Canada, we found that decision-makers operating MICs want to balance a variety of objectives, including total vaccinations, patient wait times, overall operating cost and cost per vaccine administered, and infection prevention within their facilities. The first two objectives were of greatest concern. Wait times during the period of peak demand for vaccines sometimes exceeded $2 \mathrm{~h}$ in many parts of Canada (Yang, 2009) and the United States (Las Vegas Review-Journal, 2009; NY1 News, 2009).

Cost considerations are also important from both a short-term and long-term perspective. In the short term, MIC managers would benefit from knowing the marginal productivity of labour (MPL) - that is, the number of additional vaccinations achieved from adding or subtracting one $\mathrm{HCW}$ - and whether the MPL is the same in both directions, and how much it might vary when other events occur, such as surges in demand. The MPL is important for making economic decisions about whether to hire additional staff or allocate existing staff to other facilities.

The average cost to vaccinate a patient also factors into the ongoing debate about the cost-effectiveness of mass vaccination as a response to pandemic influenza. Mass immunization programmes in Canada and the United States during the 2009 H1N1 pandemic were criticized as expensive and excessive (Amico, 2009; Waldie and Alphonso, 2009; Blackwell, 2010), with total costs in Canada and the US borne by all levels of 
government being estimated at CAD $\$ 1$ billion (Blackwell, 2010) and USD\$6.15 billion (Amico, 2009), respectively, though subsequent cost-effectiveness studies have attempted to address these concerns (Sander et al, 2010; Durbin et al, 2011). This study does not examine the overall cost-effectiveness of mass vaccination, which depends on many factors other than the effective operation of MICs.

Infection prevention at healthcare facilities is a priority throughout the sector (Centers for Disease Control and Prevention, 2010; World Health Organization, 2012). During a pandemic, infections can occur at any venue where people gather-hospitals, schools, workplaces, shopping centres, etc. The same holds true for MICs. Public health units in Ontario took care to communicate to the public that people experiencing influenza-like symptoms should not attend MICs, and the two public health units consulted in this study believed that the risk of influenza transmission at MICs from symptomatic patients was very low. However, patients with asymptomatic or very mild infections, or who are in the pre-symptom incubation period, which may last 1-4 days (Centers for Disease Control and Prevention, 2010), are believed to be infectious, albeit less so than patients with strong symptoms (Influenza Team, European Centre for Disease Prevention and Control, 2007). Reducing patient wait times has the additional benefit of further reducing the risk of influenza transmission within MICs.

This study uses a discrete-event simulation (DES) of an MIC to provide public health units with information about the likely impact of changes in staffing levels on these various measures of MIC performance. A range of scenarios are considered through a full-factorial designed experiment, including surges in demand, restrictions on vaccine eligibility, and extension of operating hours. Sensitivity analysis (SA) is conducted on several parameters that were not tested in the designed experiment.

The MIC model was built using data from MICs run by NRPH in Ontario, Canada, during the 2009 H1N1 pandemic. NRPH's MICs were run similarly to many of the large urban MICs run elsewhere in Ontario. Although some aspects of the MIC model may not overlap completely with MICs from other jurisdictions, the direction and approximate magnitude of several important effects reported in this study are likely generalizable.

\section{Literature review}

Several researchers have used DES to help plan the mass distribution of antibiotics and vaccines. Hupert et al (2002) developed a model to inform staffing decisions at a mass antibiotic distribution centre to respond to bioterrorism threats, and Washington et al (2005) developed a model to optimize staffing assignments at a hypothetical smallpox mass vaccination clinic. Both studies had to rely on hypothetical inputs and expert opinion since no such clinics have been operated.
The potential value of simulation studies to emergency vaccination planning is further discussed by Asllani et al (2007). Although the authors do not present simulation results, they do discuss the differences between how responses to diseases such as seasonal flu, pandemic flu, smallpox, and anthrax should be modelled. Because pandemic influenza can be expected to affect a larger proportion of the population than an anthrax bioterrorism event, and often spreads faster than seasonal flu, simulation studies for other vaccine or antibiotic distribution problems might not adequately reflect the highvolume and high-speed operations that MICs for pandemic influenza need to achieve (Asllani et al, 2007).

Washington (2009) examined the cost and capabilities of a mass influenza and pneumococcal vaccination clinic using simulation, and made efforts to base the model off data from a real clinic's operations. Although the model's goal was to simulate a full-capacity response-what might be expected during a pandemic - the clinic that the model was based on was operated during the seasonal flu season and had turnout that was under $50 \%$ of the targeted capacity. Andress (2003) and Phillips and Williamson (2005) discuss several insights on MIC management arising from actual MIC operations, but do not specifically address how to optimize staffing decisions.

Simulations have been used extensively to understand the dynamics of influenza spread at the international (Khan et al, 2011), regional (Los Alamos National Laboratory, 2006; Stroud et al, 2007; Savachkin and Andres Uribe-Sanchez, 2012), and municipal levels (Elveback et al, 1976; Haber et al, 2007; Das et al, 2008; Gojovic et al, 2009; Sander et al, 2010; Lee et al, 2010; Aleman et al, 2011). However, no studies, simulationbased or otherwise, have been conducted on influenza transmission risks specifically within MICs.

There have been many studies examining seasonal and pandemic influenza transmission risk in various settings, including households (Viboud et al, 2004; Sikora et al, 2010), trains (Cui et al, 2011), airplanes (Moser et al, 1979; Klontz et al, 1989; Foxwell et al, 2011; Khan et al, 2011), cars (Knibbs et al, 2012), schools (Lessler et al, 2009), universities (Mei et al, 2010; Araz et al, 2011), and hospitals (Salgado et al, 2002; Noakes and Sleigh, 2009; Barnes et al, 2010; Wong et al, 2010; Bearden et al, 2012). For the most part, these studies document particular outbreaks and discuss context-specific risk factors and infection control measures, and do not generalize well to MICs. For example, whereas patients at a busy MIC may spend up to $2 \mathrm{~h}$ at the facility and may come into proximity with a dozen or so other patients and 2-3 HCWs, a hospital inpatient may spend days in a hospital, making contact with a greater number of HCWs, possibly less contact per day with other patients, but also more contact with physical objects and surfaces in the facility. Furthermore, whereas MICs are for patients without symptoms of influenza, hospitals may house patients with acute influenza, as well as patients who are more susceptible to the virus.

The modes and magnitude of pandemic influenza transmission risk at MICs likely differ from those of other healthcare 
facilities. The techniques this study uses to estimate this risk are adapted from widely used methods in agent-based pandemic modelling at the regional and municipal levels (Elveback et al, 1976; Los Alamos National Laboratory, 2006; Haber et al, 2007; Stroud et al, 2007; Das et al, 2008; Aleman et al, 2011; Savachkin and Andres Uribe-Sanchez, 2012). These methods compute individual infection probabilities based on a susceptible person's exposure time to infected persons in different subenvironments (eg households, workplaces, schools), taking into account heterogeneous mixing patterns, and sometimes adjusted for factors that are believed to increase an individual's susceptibility or infectivity. All of these simulations work in hourly or daily time-steps. To offer a more granular picture of potential transmission risk at MICs, this study simulates and records susceptible patient interactions with infectious patients by the second.

This study is the first to simulate a pandemic influenza MIC using data from real clinics operated during a pandemic. The study builds upon the MIC management literature by considering a more complete range of facility performance measures and by testing the impact of staffing changes under different scenarios. It is also the first study to model the risk of influenza infection at an MIC during a pandemic, and to use such risk measures to help inform staffing decisions.

\section{Model overview}

The flow of patients through an MIC involves a modest number of decision points, queues, and processes, as illustrated in Figure 1. NRPH pioneered an electronic patient record system that was used throughout Ontario in 2009 to manage patients and record service-time data at MICs during the $\mathrm{H} 1 \mathrm{~N} 1$ pandemic. The system recorded a timestamp for every instant that a patient's record was accessed or closed at a service station, corresponding in theory with their physical arrival and departure from the service station.
Data from this electronic system were obtained for 26-28 October 2009 at one facility, coinciding with the first three days of the public immunization programme. Data for 3, 6, and 12 November at a separate facility were also used, coinciding with a provincial policy that restricted vaccine access to prioritized groups. The combined data sets provided arrival and service time records for 9598 patients. The pandemic in Canada is considered to have peaked in early November (Public Health Agency of Canada, 2010). In addition, hourly staffing schedules and the number of patients seen per hour per staff member were also provided, along with staffing costs, floor plans, and facility operation instructions.

The input data from NRPH revealed several complex patterns that could not be captured with analytical queuing models. Patient arrival rates and service times at registration and injection stations varied according to the hour of the day, the age of the patient, and the size of the family group with whom patients arrived. It was also found that service times decreased as wait times increased. We conjecture that MIC staff could have increased their productivity in response to seeing long queues build up. Furthermore, the presence of prioritization policies that restricted vaccine access to certain groups based on age and health status had an impact not only on the total patient turnout, but also on the size of arriving groups and the patient age distribution.

The parameters used in the baseline scenario for the model are based on the staffing levels and patient demand experienced on 26 October 2009 at a particular MIC which was described by $\mathrm{NRPH}$ as operating at full capacity. The remainder of this section describes the model's components in greater detail.

\subsection{Patient arrival rates}

Patients who arrive at the MIC must wait outside if the facility is not yet open or at capacity. Patients arrive in batches ranging in size from one individual to families of five, starting $30 \mathrm{~min}$ before the official facility opening time at 8:30 am.

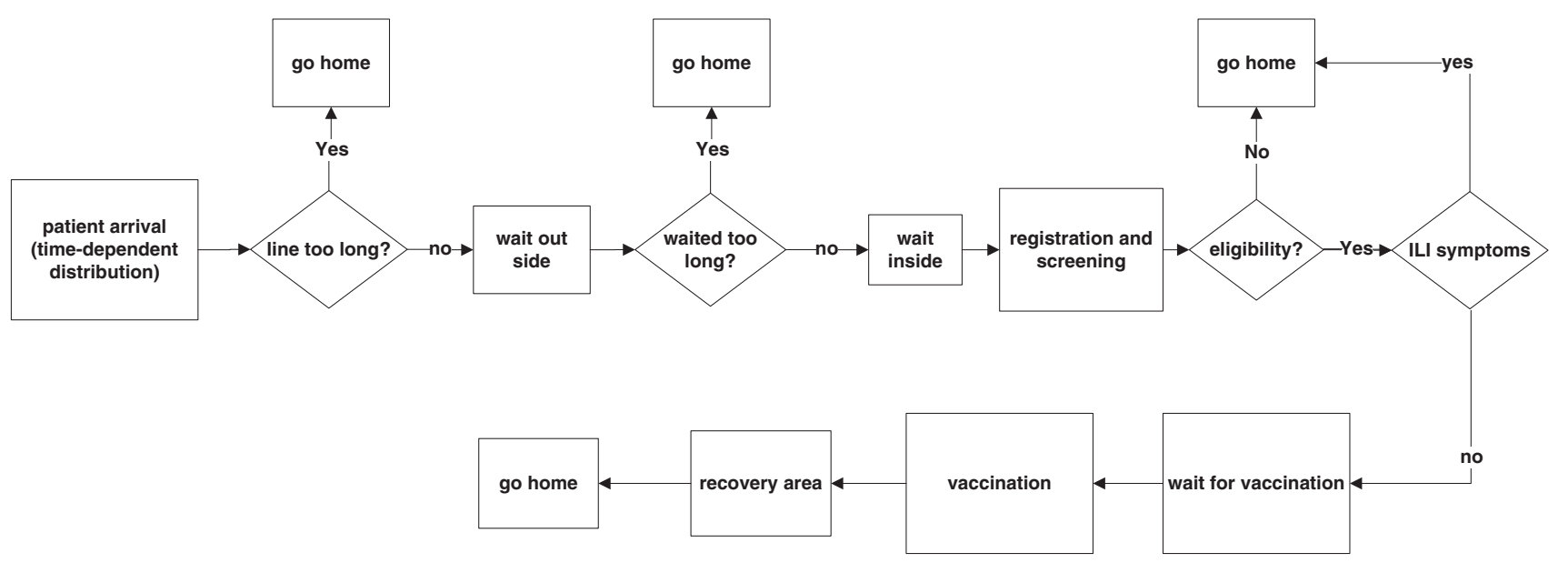

Figure 1 Flowchart of patient movement through MIC in Niagara Region, ON. 
The distribution of family batch sizes was provided by NRPH (Gentry, 2011). No incidences of families with six or more members arriving together were recorded. The arrival of family groups was modelled as a non-homogeneous Poisson process, with interarrival times being exponentially distributed. Arrivals were set to occur until 6:30 pm in a full-capacity scenario and 7:00 pm in a hypothetical overloaded scenario, marking the time that facility doors would close to new patients.

Patient arrival rates had to be estimated indirectly because patients were only recorded in the electronic record system once they reached the registration desk, after arriving and waiting in line. The arrival rates were calibrated to ensure consistency with several other known parameters: the total number of patients vaccinated, the maximum pre-registration wait time noted by staff, and the wait times and patient queue lengths within the facility between service stations. Differences existed in patient turnout between days and between facilities, requiring separate arrival rate calibrations to replicate each day's turnout. The baseline scenario used in this study reflects turnout and staffing levels at an NRPH clinic on 26 October 2009.

NRPH reported that patient arrival rates varied by the hour. Although patient arrival times were not recorded directly, NRPH's data show hourly variations in staff productivity that seemed consistent with the manager's description of the hours that were typically busiest (mid-afternoon and early evening). The interarrival times used for the 26 October baseline scenario are presented in Figure 2.

\subsection{Patient characteristics}

The patients are assigned labels indicating their health status, their age group, whether they belong to a government-defined priority group, the size of the group with which they arrived, and whether they have eligible provincial health insurance. Data collected by NRPH were used to determine the proportion of patients assigned to each category.

Each patient is assigned one of two health states: susceptible or infectious. Public health officials in the Niagara Region reported that public communications efforts informing people not to come to MICs if they had influenza-like symptoms were

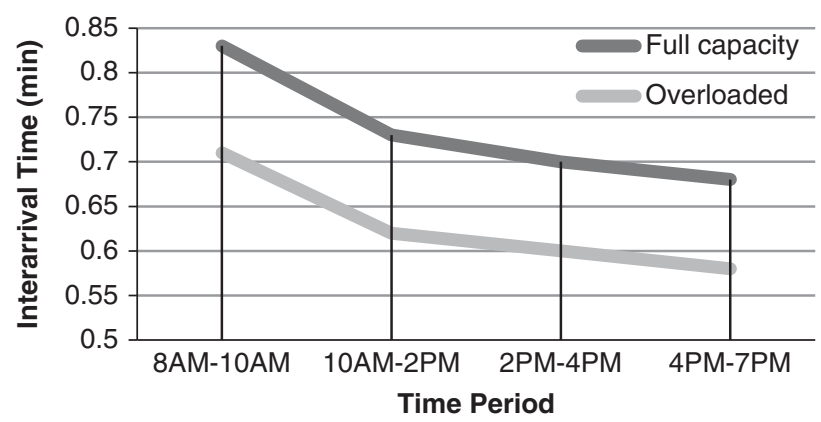

Figure 2 Interarrival times in minutes for patient batches in two scenarios. largely successful. Consequently, the model assumes that infectious patients at the MIC were asymptomatic or had mild enough symptoms to prevent effective identification and screening. The simulation was run with 0,2 , and $3 \%$ of arriving patients being infectious, with the remainder of patients being susceptible.

Since transmission risk is believed to be age-dependent (Haber et al, 2007), patients were assigned to one of four age groups: $0-4,5-18,19-64$, and $65+$ years of age. The service times at registration and injection stations were also found to be age-dependent. The percentage of patients belonging to each age group depended on the government-mandated prioritization restrictions in place. Prior to 31 October 2009, no prioritization restrictions were in place. From 1-13 November, access to vaccines was restricted to the following groups: people under 65 with chronic medical conditions; pregnant women; children six months to five years in age; healthcare workers involved in pandemic response or the delivery of essential healthcare services; and care providers or household contacts of people at high risk who cannot be immunized. From 14-16 November, the restrictions were relaxed to include youth up to the age of 18 , household members of pregnant women, first responders, and provincial corrections officers; however, data on the distribution of ages and group arrival sizes for this period were not available. From 17 November onwards, vaccine access was unrestricted. Table 1 contains age-group patient percentages for the initial unrestricted period and the first stage of restricted access.

NRPH's data indicate that expected service times at registration and injection stations also depend on the size of the patient's family group. The likelihood of a patient belonging to a family group of a particular size receiving treatment on the same day is summarized in Table 2.

Table 1 Percentage of patients per age group, by vaccine priority restriction scenario (Statistics Canada, 2006; Gentry, 2010)

\begin{tabular}{lrrrr}
\hline & \multicolumn{4}{c}{ Age range (years) } \\
\cline { 2 - 5 } & $0-4$ & $5-18$ & $19-64$ & $65+$ \\
\hline Canadian population & 5.84 & 18.06 & 63.38 & 12.72 \\
Unrestricted access, 26-31 October & 14.61 & 17.35 & 59.58 & 8.46 \\
Restricted access, 1-13 November & 16.58 & 16.32 & 61.16 & 5.94 \\
\hline
\end{tabular}

Table 2 Percentage of patients per family batch size, by vaccine priority restriction scenario (Gentry, 2010)

\begin{tabular}{lcccrc}
\hline & \multicolumn{5}{c}{ Family size } \\
& 1 & 2 & \multicolumn{1}{c}{3} & \multicolumn{1}{c}{4} & 5 \\
\hline Niagara population & 26.0 & 31.0 & 20.0 & 14.0 & 9.0 \\
Unrestricted access, 26-31 October & 35.0 & 28.0 & 20.0 & 13.0 & 4.0 \\
Restricted access, 1-13 November & 52.5 & 27.5 & 13.0 & 6.0 & 1.0 \\
\hline
\end{tabular}




\subsection{Patient wait-time tolerance, line tolerance, and facility closure}

It is possible that some patients may balk at the size of the MIC's line and choose to leave, while other patients may reneg on their decision to get vaccinated if they have waited too long. Balking and reneging are well-established as phenomena in many queuing systems, including healthcare settings (Zenios, 1999; Fomundam and Herrmann, 2007; Asaro et al, 2008). However, the factors that can influence balking and reneging rates, such as the value and substitutability of the service, the possibility of postponement, and the customer's cost of waiting, vary highly across contexts. No empirical estimates for reneging and balking rates for mass immunization or sufficiently comparable health service settings have been published. Unfortunately, it was not possible to observe reneging and balking behaviour directly because the MICs could only record patients who made it to the registration desk.

The possibility of reneging and balking was built into the model to allow realistic bounds to be placed on outdoor wait times. These bounds were deemed particularly important for simulated scenarios in which there would be staff shortages and significant queue build-up. It seemed necessary to generate a bound of sorts since patients would have had a limited willingness to wait. Although reneging may be less common in healthcare settings where there are steep health consequences for postponing/cancelling service, or significant fines for missed appointments, MIC reneging does not impose such costs on patients. Since the particular reneging parameters used were informed guesses and not grounded in data, detailed SA on the reneging and balking parameters is presented in the Results Section. Varying the reneging and balking parameters did not have a significant effect on total vaccinations, though outdoor wait times were affected.

For reneging, a patient's maximum tolerance for waiting outside the facility before leaving altogether was generated using a normal distribution with a mean of $90 \mathrm{~min}$ and a standard deviation of $20 \mathrm{~min}$. For balking behaviour, the simulation gives patients a line length tolerance: if a newly arrived patient finds the number of people in line waiting to enter the facility is greater than their line tolerance, they will simply go home. Intuitively, a person's line tolerance and waittime tolerance are likely correlated. Under this assumption, the line tolerance was set to be normally distributed with a mean of twice the wait-time tolerance level and a standard deviation of $20 \mathrm{~min}$. An hour before the facility closes at 6:30 pm, the MIC stops taking new patients and closes its doors. Patients still outside at this time go home while the patients inside proceed through the MIC.

\subsection{Patient screening}

It was assumed that at least $0.5 \%$ of patients arriving at the facility would not be eligible to receive a vaccination by virtue of not holding provincial health insurance or not belonging to an eligible priority group. In the model, such patients are sent home after being screened at the registration desks. Patients with a health state involving symptoms of influenza-like-illness (ILI) are also screened at this stage. The effectiveness of screening can be set to less than $100 \%$ to reflect the possibility that some patients with mild ILI symptoms may want vaccination and consequently claim to be feeling fine to avoid being sent away. The number of patients turned away for these reasons at actual MICs in the Niagara Region is not known.

\subsection{Registration and vaccination stations}

Once inside the MIC, patients enter the registration queue, which has a 50-person capacity. At capacity, patients are unable to enter the facility and wait outside. At registration, eligible patients provide clerical staff with personal data and answer questions about co-morbidities and possible contra-indications. Following registration, patients enter a new queue to receive the vaccine from nursing staff. The queue for vaccine administration has a maximum capacity of 250 to reflect space constraints. When this queue reaches capacity, the registration desks are programmed to stop processing patients until space becomes available. After being vaccinated, patients wait in a recovery area for $10 \mathrm{~min}$ in case they have an adverse reaction to the vaccine. Then, patients leave the facility.

Each point of service-registration, flu assessment, and vaccination-is assigned a distribution for service times, as well as a certain number of staff, each of whom serves one patient at a time. Two data sets were used to generate the service time distributions. The first data set consisted of entries from NRPH's electronic patient record system that captured the length of time that a patient's electronic file was open at each service station. The second data set contained the number of patients served by each staff member over each hour of the day. The service times recorded in the electronic system were found to be significantly lower than those implied by the hourly throughput and the overall number of patients vaccinated by the facility, even when taking into account break time and minor inefficiencies. Consequently, the mean service time was estimated using the number of patients seen per hour per staff member. The service times recorded in the electronic patient system were still used to obtain standard deviation estimates, to infer a likely shape of service time distributions, and to estimate the effect of patient age on service time.

The sample standard deviation of the registration times from 26 to 28 October 2009 was $1.16 \mathrm{~min}$, and $1.25 \mathrm{~min}$ for vaccination times. The distribution of service times in the electronic record system at both registration and vaccination stations was positively skewed (Figure 3), and, of course, nonnegative. Lognormal distributions were chosen to generate both registration and vaccination times so as to replicate this positive skew and non-negativity.

The effects of patient age group on service time was found through fitting an ANOVA model, and are summarized in Table 3. All effects are relative to the $0-4$ age group. The data 


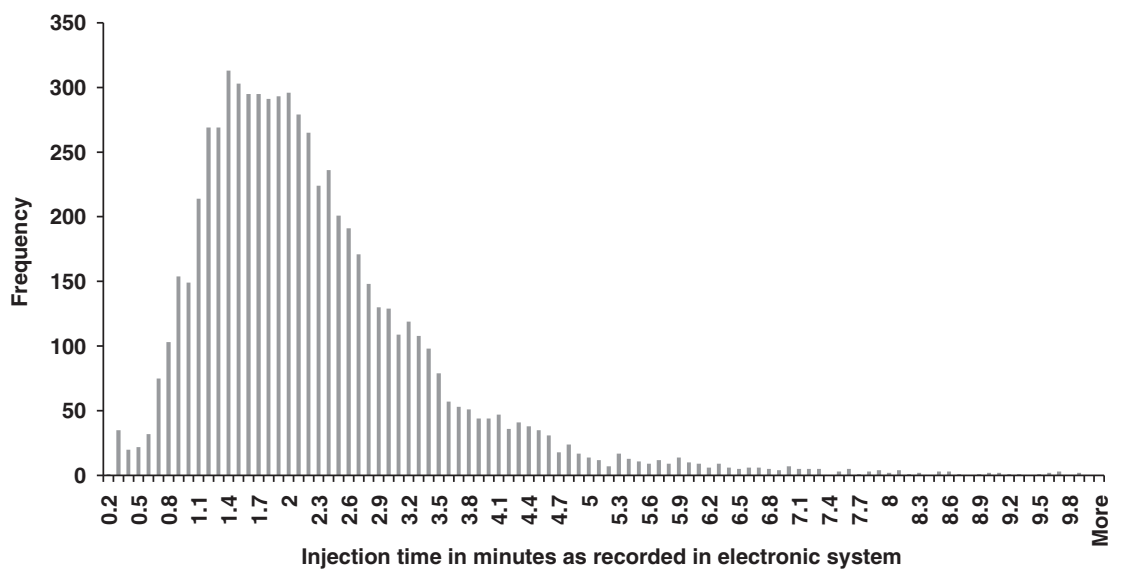

Figure 3 Histogram showing positive skew for injection times recorded in electronic system, 26-28 October 2009.

Table 3 Effects of patient age on service time (minutes), relative to service times for the $0-4$ age group

\begin{tabular}{llrlrr}
\hline \multirow{2}{*}{ Age range } & \multicolumn{2}{c}{ Registration } & & \multicolumn{2}{c}{ Vaccination } \\
\cline { 2 - 3 } \cline { 6 - 6 } & Effect & p-value & & Effect & $p$-value \\
\hline $5-18$ & 0.047 & 0.043 & & -0.22 & 0.050 \\
$19-64$ & 0.550 & $<0.001$ & & -0.40 & $<0.001$ \\
$65+$ & 0.760 & $<0.001$ & & -0.30 & $<0.001$ \\
\hline
\end{tabular}

suggest that adults and seniors take roughly 32 and $45 \mathrm{~s}$ longer to register than small children, respectively, perhaps because they are more likely to have more complicated medication and health histories to document at the registration desk. The data also show that children under 5 years require slightly more time to vaccinate than older patients. These findings mean that the optimal mix of registration staff and vaccination staff could change depending on the demographic profile of the patient population. Based on these numbers, if 1000 single adults attending a large MIC were substituted with additional children in the family batches, 8.9 fewer registration clerk hours and 6.7 additional nurse hours would be required to meet demand, all else held equal. Since vaccine prioritization rules are often based on age, major changes in patient demographics are quite likely and may affect staff planning.

In addition to age-based adjustments, the expected value of a patient's registration time also depends on the size of the family group with whom she/he arrived (Gentry, 2011), since address information and family name spelling might not need to be repeated for additional family members. Initial estimates for these service time differences were based on the registration time-stamps from the electronic patient record system. However, the service times recorded electronically seemed to represent only a quarter of the time registration staff spent at the registration desk registering patients based on our analysis of NRPH's staffing data. Consequently, the expected registration times used in the simulation were set to four times the
Table 4 Effects of family group arrival size on registration time per-patient

\begin{tabular}{lccccc}
\hline Family batch size & 1 & 2 & 3 & 4 & 5 \\
\hline Expected registration time (min) & 3.60 & 3.48 & 3.25 & 3.18 & 3.28 \\
\hline
\end{tabular}

average values calculated using the electronic records, and are presented in Table 4. The weighted average of these registration times ranges from 3.42 to 3.49 min, depending on which prioritization policy is in place, and is close to the rate of $3.45 \mathrm{~min}$ calculated indirectly by dividing total minutes believed to have been worked by the total patients registered over those days.

Vaccination times did not vary with the patient's family size. However, the data sets did reveal a difference in productivity between nurses with different professional specialties. In particular, vaccine preventable disease nurses and infectious disease nurses, who represented just under half the nurses at the MICs, served patients about $12 \%$ faster than other nurses ( $p=0.0109$ ), though this effect was only statistically significant for the earliest two of the six days for which data were provided.

\subsection{Changes in staff productivity by hour}

Although the MIC managers believed there to be a consistent stream of patients served by staff working at full capacity, the data sets revealed significant variation in staff productivity over different hours of the day, across different days, and among staff members. Although productivity on 26 October for both nurses and registration staff was lowest in the morning and highest in the $2 \mathrm{~h}$ before closure, the variation on 27 October did not exhibit a clear trend, while productivity on 28 October was highest in the early afternoon, as shown in Figure 4. The extent of the variation is significant, but its causes are unclear.

The differences among staff were also quite high, even though the MIC managers described the 26-28 October 2009 period as especially busy, with staff working non-stop and very 


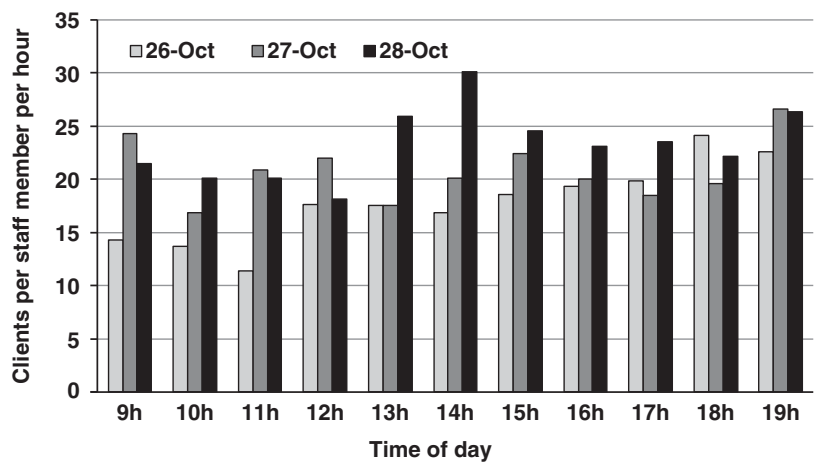

Figure 4 Registration staff productivity by time of day.

hard. For example, the standard deviation of each registration staff member's average hourly productivity during the unrestricted vaccine access stage was 5.5 patients per hour, or just over $25 \%$ of the average rate of 20.0 patients registered per hour. Variation between nurses at vaccination stations was just as large. On most days, the most productive nurses and registration staff served twice as many patients per hour as their counterparts with the lowest productivity. When compared with the most productive hour worked by any staff member on a given day, average staff productivity was only $46-56 \%$ of the top performer's best hour. This gap has two components: staff members' average hourly productivity was $81-85 \%$ of their own personal bests for the day; and the averages of the daily personal bests were only $58-70 \%$ the level of the top performer's personal best. Figure 5 shows how average hourly productivity and average personal best productivity of registration staff varied over three consecutive days during the busiest period, while falling in the month of November, in part due to lower MIC utilization.

The number of people visibly waiting could have put greater pressure on staff members, leading to a faster work pace. However, the data sets did not contain information on outdoor queue lengths at different times of the day, so this hypothesis could not be verified. It is not known whether small errands may have disrupted some nurses assigned to vaccination stations. Such disruptions could bias these productivity estimates downwards, while upwardly biasing the productivity variance.

Since an effect of queue length on service time seemed plausible, the service times for a patient in the simulation were reduced by $4.8 \mathrm{~s}$ for every 10 people waiting behind them in line, inside or outside the facility. This adjustment helped the simulation replicate some of the productivity changes observed over each day, with faster service times coinciding with periods when the lines were longest. It is not known whether this positive relationship between line length and service times was present at other facilities.

\subsection{Operating costs}

To calculate the average cost per vaccine administered, labour costs for registration clerks and nurses, vaccine material costs,

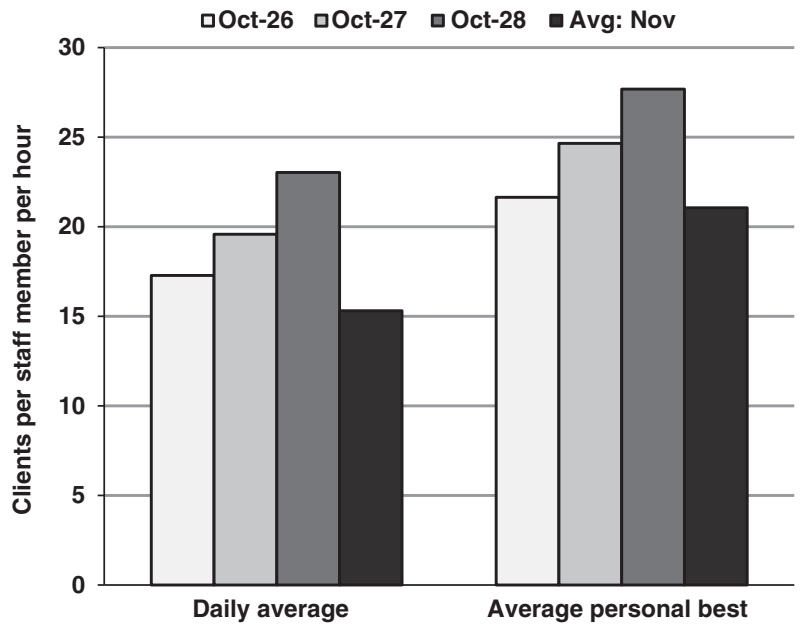

Figure 5 Comparison of hourly productivity, 26-28 October and November.

Table 5 MIC operating costs (CAD)

\begin{tabular}{lll}
\hline Item & Cost & Comments \\
\hline Vaccine cost per unit & $\$ 10$ & $\begin{array}{l}\text { Government purchase price (CBC } \\
\text { News, 2009)+ shipping }\end{array}$ \\
$\begin{array}{l}\text { Daily space rental } \\
\begin{array}{l}\text { Hourly nurse wage } \\
\text { plus benefits }\end{array}\end{array}$ & $\$ 500$ & $\begin{array}{l}\text { N/A for NRPH's MICs } \\
\text { Actual (Gentry, 2011) }\end{array}$ \\
$\begin{array}{l}\text { Hourly clerk wage } \\
\text { plus benefits }\end{array}$ & $\$ 27$ & Actual (Gentry, 2011) \\
\hline
\end{tabular}

and space rental costs were included in the model (Table 5). The cost of administration, information technology, and greeters was not included, so the overall cost per vaccine in the model is conservative. The space rental costs is an estimate of what a public health unit would have to pay in the event that a free public facility is not available.

\subsection{Risk of disease transmission}

Influenza transmission is believed to be unlikely at distances greater than $2 \mathrm{~m}$ from an infected person (Brankston et al, 2007). The risk of a contagious patient infecting a susceptible patient is modelled using the function $1-e^{-\lambda_{i j} t}$ (the cumulative density function of an exponential distribution), where $t$ is the amount of time the two patients spend within $2 \mathrm{~m}$ of each other, and where $\lambda_{i j}$ is the age-dependent hazard rate (probability of infection) for a minute of exposure between susceptible person $i$ and infectious person $j$, given their respective age groups. The difference in transmission risk for asymptomatic, pre-symptomatic, and mild-symptom cases compared with fully symptomatic cases is not known, but believed to be less severe (Influenza Team, European Centre for Disease Prevention and Control, 2007). To err on the side of caution, the transmission risk rates used in the MIC simulation represent average 
Table 6 Pandemic influenza transmission hazard rates $(\lambda)$ per minute (Haber et al, 2007)

\begin{tabular}{lcccc}
\hline $\begin{array}{l}\text { Age group of } \\
\text { infectious person }\end{array}$ & \multicolumn{4}{c}{ Age group of susceptible person } \\
\cline { 2 - 5 } & $0-4$ & $5-18$ & $19-64$ & $64+$ \\
\hline $0-4$ & 0.00059 & 0.00062 & 0.00033 & 0.00080 \\
$5-18$ & 0.00058 & 0.00061 & 0.00033 & 0.00080 \\
$19-64$ & 0.00057 & 0.00053 & 0.00032 & 0.00080 \\
$64+$ & 0.00057 & 0.00054 & 0.00029 & 0.00102 \\
\hline
\end{tabular}

transmissibility calibrated in the population-level influenza simulation study by Haber et al (2007). These rates are given in Table 6.

Susceptible patients can be exposed to multiple infectious persons simultaneously. The patient's hazard rate for infection at a given point in time is set to the sum of the individual hazard rates for all of the infectious persons with whom they are making contact at that point in time. Since the number and type of infectious persons a susceptible person may be exposed to will change as she/he progresses through the MIC, the simulation re-assesses with whom the susceptible person has contact every time his/her position in the MIC changes.

To track total exposure time to contagious patients, each susceptible person is assigned an exposure variable that gets updated at every time-step $k$. To update the exposure variable, the simulation must determine which individuals are within $2 \mathrm{~m}$ of a given susceptible individual, and then check their health status. For computational speed, the 2-m radius was assumed to include individuals within $M$ ranks on either side of the individual's position in a queue during time $t_{k}$. It was assumed that $M=3$ for queues inside the MIC and $M=4$ for queues outside the MIC entrance. The maximum number of people with whom a patient can have contact at a given time is therefore $2 M$. To represent greater congestion or adjacent single-file queues, $M$ can be increased appropriately.

Letting $\mathcal{K}_{i}$ represent all time intervals $k$ over which susceptible person $i$ occupies a distinct position in the MIC system, and letting the set $\mathcal{I}_{i k}$ represent all infectious patients with whom the susceptible person has contact during time period $t_{k}$, the probability of a susceptible person $i$ becoming infected in the MIC can be represented as $1-e^{-\sum_{k \in \kappa_{i}} \sum_{j \in \mathcal{I}_{i k}} \lambda_{i j} t_{k}}$.

The expected number of infections per day is the sum of the probabilities of infection for each susceptible individual in the system that day. The simulation does not consider the probability of transmission to HCWs on duty since the transmission risk to individuals wearing protective masks is likely low, and it is likely that most MIC workers would be vaccinated.

In very long queues, the people to whom a susceptible person is exposed is re-assessed every time the person's rank in the queue changes, even though this set of people may remain constant for up to $60 \mathrm{~min}$. This superfluous calculation can be rectified by simply multiplying the sum of the hazard rates from those same individuals by the time interval over which the patient is in queue with them. However, even without this reduction in computations, the model's one-day run time is under $90 \mathrm{~s}$

\section{Model validation}

It is not possible to validate the estimates of the number of patients becoming infected at an MIC against empirical data on infection risks in MICs or in other public venues because no such empirical data exist.

The infection risk parameters used in this simulation were originally generated for use in a population-level influenza spread model (Haber et al, 2007) so as to ensure that the proportions of the simulated population in different age groups that became infected would resemble the proportions from past pandemics. It is important to recognize that all infection risk parameters calculated in this way are limited for a number of reasons: the set of parameter values that could lead to the desired simulated pandemic outcomes is not necessarily unique; the infection risk parameters depend on the assumptions made about social contact patterns, which change by culture, geography, and socioeconomic conditions; infection risk is affected by ambient conditions such as relative humidity and temperature (Tellier, 2006); and there is no consensus on which physical mechanisms of transmission are most important (Tellier, 2006). In light of these limitations, the infection counts produced by this simulation model should be viewed as ballpark estimates, subject to revision as more becomes known about the probability of influenza transmission at the individual level.

Validation for the overall design of the model was done by setting staffing and arrival rate levels to match those of the actual MICs for which NRPH provided data, and comparing the empirical patient count, post-registration wait times, and indoor queue lengths with those generated by the simulation. In order to ensure that the simulation's outputs were within $10 \%$ of the empirical outputs, it was necessary to reduce nursing staff availability by $5 \%$, that is, nurses would be at their vaccination stations only $95 \%$ of the time, not counting official breaks. This change may reflect the fact that nurses sometimes had to serve other functions in the MIC, such as collecting vaccination supplies from storage and distributing them to other nurses.

\section{Results}

The simulation model was built using Simul8 (Visual8, Mississauga, $\mathrm{ON}$ ) and run on a Windows 7 computer with a 2.1 $\mathrm{GHz}$ processor and 4GB of RAM. The peak-capacity (baseline) scenario and several hypothetical scenarios were simulated to understand the relationship of infection risk, throughput, and costs

From an economic point of view, the results offer strong reasons to invest additional resources in MICs in order to reduce wait times. Shorter wait times also mean less exposure 
to contagious patients - an additional public health benefit. A designed experiment was performed to examine how changes in a variety of factors affect several measures of MIC performance.

\subsection{Baseline scenario}

An 'as-is' baseline configuration of the MIC model was created based on the staffing levels, patient turnout, and hours of operation of an MIC operated by NRPH on 26 October 2009, described by management as operating at full capacity. On this day, no restrictions were in place on who could access vaccines. Nine registration staff and 11 vaccination nurses (four VPD/ID RNs and seven regular RNs) were on duty throughout the day. The facility opened its doors at 8:30 am and closed them at 6:30 $\mathrm{pm}$, at which point it would only serve patients already inside the facility. Two per cent of patients were assumed to be infectious and without symptoms. The response variables tracked in the simulation and their results are presented in Table 7. Patients with influenza but no symptoms were not included in the number of people vaccinated or in the cost per vaccination calculation since their vaccinations would not benefit them.

The trials show that the expected number of infections occurring in the MIC is roughly $0.55 \%$ of the number of people vaccinated. The count is still high enough to merit attention to transmission risk in MICs, but does not detract from the benefits of mass immunization. The average number of patients registered per full-day clerk was 184.3 , and the average number vaccinated per full-day nurse was 150.8 .

Although patients spent close to $91 \mathrm{~min}$ on average at the MIC, less than $10 \%$ of that time was spent receiving services from MIC staff. Most of the time was spent waiting, and a large part of that wait time took place outside. The total number of person-hours spent by the public at the MIC, including people who did not get into the facility in time or who were screened on the basis of eligibility, was 2644.9 , with a $95 \%$ confidence interval of $(2516.9,2772.9)$. Patients who arrived and then left immediately because the line length exceeded their line tolerance were excluded from the average time in system (ATS) calculations.

It would be unrealistic not to assign some value to this lost time, which ultimately does impose a cost on the economy and

Table 7 Baseline scenario results, 21 replications

\begin{tabular}{lcc}
\hline Response variable & Value & $\begin{array}{c}\text { 95\% confidence } \\
\text { interval }\end{array}$ \\
\hline $\begin{array}{l}\text { Expected infections } \\
\text { Number vaccinated }\end{array}$ & 9.08 & $(8.54,9.63)$ \\
$\begin{array}{l}\text { Direct cost per vaccination (CAD) } \\
\text { Societal cost per vaccination }\end{array}$ & 165.76 & $(1645.5,1672.2)$ \\
(CAD) & 32.32 & $(31.94,32.70)$ \\
$\begin{array}{l}\text { ATS-Average time in system } \\
\text { (min) }\end{array}$ & 90.89 & $(88.87,92.91)$ \\
\hline
\end{tabular}

society, directly by taking up formal work time, or indirectly taking away time from domestic activities. Wait times were penalized with a cost of $\$ 10$ per hour, roughly Ontario's Minimum Wage in late 2009, to allow us to estimate the total social cost per vaccination, comprising of both lost time and MIC expenditures. Taking wait times into consideration in this way roughly doubled the cost per vaccination from $\$ 16.76$ to $\$ 32.32$.

\subsection{Sensitivity analysis}

Since the number of distinct scenarios to test in a full-factorial designed experiment grows exponentially in the number of factors, it was not possible to test the simulation for all possible sources of variation via the experiment. To compensate, oneway SA was conducted on several uncertain parameters not tested in the experiment. The results for response variables of interest are presented.

5.2.1. Patient interarrival times. The distribution of patient arrival times in the baseline scenario was chosen to ensure that the number of persons receiving vaccination and the ATS were consistent with the values for the reference clinic that the baseline scenario is supposed to imitate. The reference clinic served 1699 patients on 26 October 2009. Since the model only counts vaccines given to the $98 \%$ of patients who are uninfected as useful vaccinations, the arrival rates were calibrated to yield an average vaccination count close to $0.98 \times$ $1699=1665$. Patient interarrival times also impacted ATS. The target ATS value range for the baseline clinic was 85-95 min, including time spent in line outside the facility prior to registration. The interarrival times for all periods of the day were varied by a multiplicative factor applied to the baseline values, with 10 replications per case. The results are presented in Figure 6.

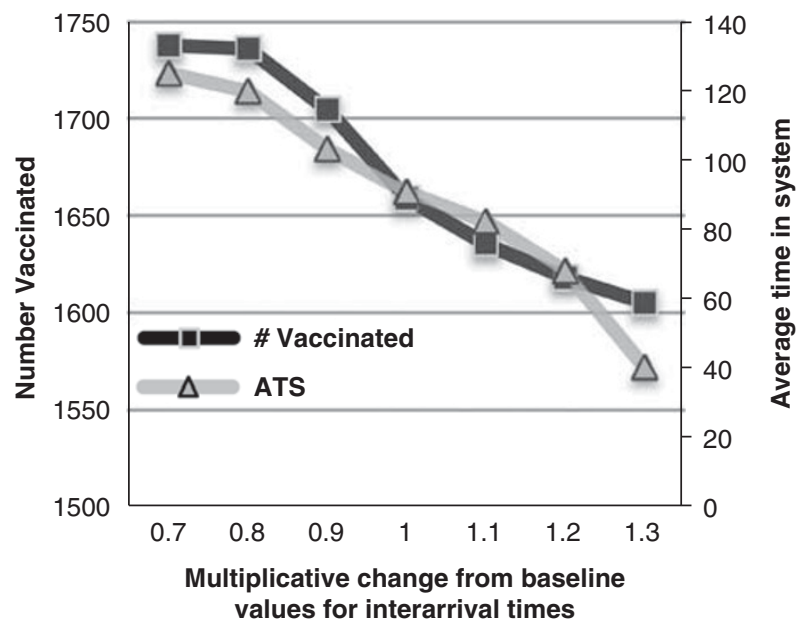

Figure 6 Vaccinations and average time in system versus patient interarrival time adjustment. 
The range of variability for total vaccinations is bounded above by the physical capacity of the facility, with small increases happening alongside faster arrival rates as a consequence of the slight relationship between visible queue lengths and staff service times assumed in the model. The total number vaccinated did not fall proportionally with the lower rates of arrival because a large portion of patients in the baseline scenario were going home prior to entering the facility because of the long outdoor queue. When the rate of arrivals was slower, the queue was shorter, resulting in a higher proportion of the arrivals staying in the queue and entering the facility-this partially compensated for the slower arrival rate. In addition, while the ATS increased with higher turnout, patient wait time tolerance limited ATS growth by causing some patients to go home without entering the MIC after having waited in the outdoor line.

5.2.2. Patient wait time tolerance. Patient wait time tolerance was also varied by using multiplicative factors, with 10 replications per case. The results are presented in Figure 7. The effect on total vaccinations was limited, but more noticeable for ATS.

5.2.3. Transmission parameters. Many factors are believed to influence the risk of influenza transmission, such as relative humidity and temperature (Tellier, 2006), ventilation (Moser et al, 1979), and intensity of symptoms (Influenza Team, European Centre for Disease Prevention and Control, 2007), though these effects have not been quantified in studies involving realistic transmission between humans. To account for this uncertainty, the hazard rate parameters for influenza transmission were varied by $50 \%$, holding all other parameters at their baseline values. Twenty replications per case were used. The mean number of infections in the lower and higher risk scenarios was 4.23 and 13.51 , with $95 \%$ confidence intervals of $(3.24,5.22)$ and $(11.80,15.22)$, respectively,

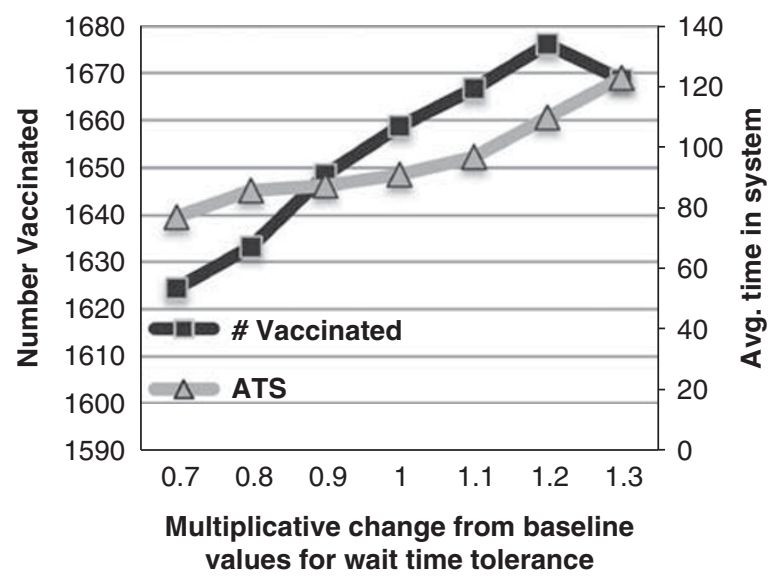

Figure 7 Vaccinations and average time in system versus patient wait time tolerance adjustment. compared with 9.08 infections in the baseline. The percentage change in the number of infections is similar to the percentage change in the transmission rates.

5.2.4. Arrival of symptomatic patients. In the baseline scenario it was assumed that patients with symptoms of influenza would not come to the MIC. Relaxing this assumption, we let an additional $1 \%$ of the population be infectious and symptomatic, and allowed for screening at the registration desk with 100,75 , and $50 \%$ efficacy. Twenty replications per case were used. The mean number of infections in these three cases was 11.20 (9.82, 12.58), 11.98 (10.83, 13.13), and 12.56 (11.57, 13.57), respectively. The increase in the number of infections, although small, was statistically significant in all cases. As the screening effectiveness decreased, infections increased slightly, but the increases were not statistically significant.

\subsection{Designed simulation experiment}

A simulation experiment was conducted to determine the effects of the six factors presented in Table 8 on total vaccines administered, ATS, expected infections, and costs per vaccination. Data from a full-factorial design with one run per scenario were combined with 20 additional replications of the baseline scenario to fit a linear ANOVA model with main effects and several interactions of interest. A total of 344 simulation runs were used in the data analysis. The results for each response variable are summarized in Table 9. Only effects that are both economically and statistically significant effects are presented. The values in the tables represent the relative difference from the baseline scenario described earlier.

5.3.1. Number of vaccinations. All factors have a statistically significant effect on the number of susceptible patients receiving vaccinations. The effect of the priority restriction policy in lowering turnout reflects the fact that these policies make it difficult for large families to arrive and get vaccinated all at once, meaning more of the arrivals will consist of individuals or couples. This decline in turnout is consistent with the decline observed in November when the priority restrictions were actually in place (Gentry, 2011).

When patient turnout was representative of the population, all response variables were affected. This factor level was included to determine whether it is reasonable to assume that the patient population is representative of the actual population without impacting the simulation's output. The differences in response variable outputs between scenarios using a representative population versus the actual population profile show that this is not the case.

Marginal changes in the number of nurses had significant effects on the number of patients vaccinated, but the size of the effect depended on other factors. In the baseline scenario, adding one nurse led to a small gain of only 50.7 new vaccinations, while removing one nurse led to a loss of 126.1 
Table 8 Factors examined in experiment

\begin{tabular}{ll}
\hline Factor & Levels \\
\hline Factor 1: Priority access restrictions & Level 1: No restriction; patient demographic is similar to actual patient in NRPH during H1N1 \\
& Level 2: No restriction; patient demographic is representative of Canadian population \\
& Level 3: Restricted to priority groups
\end{tabular}

Factor 2: Nurse staffing

Level 1: Maintain 11 nurses from baseline scenario

Level 2: Reduce the number of specialist RNs by one

Level 3: Increase the number of specialist RNs by one

Factor 3: Registration clerk staffing

Level 1: Maintain nine registration clerks from baseline scenario

Level 2: Reduce the number of clerks by one

Level 3: Increase the number of clerks by one

Factor 4: Hours of operation

Level 1: Maintain doors open at 8:30 am and close at 6:30 pm

Level 2: Doors open at 8:00 am and close at 7:00 pm

Factor 5: Infection rate

Level 1: Maintain at $2 \%$ of patients as asymptomatic infectious

Level 2: Increase to $3 \%$ of patients asymptomatic infectious

Level 3: No patients are infectious

Factor 6: Surge in patient demand

Level 1: Patient arrival rate based on 26 October 2009 turnout

Level 2: $15 \%$ surge in patient arrival rate

vaccinations. However, the benefit of adding a nurse is 28.2 vaccinations greater if an extra clerk is also added, though adding a clerk alone had no significant effect on total vaccinations, and removing a clerk led to a meagre decline in vaccinations of 39.2. The benefit of adding a nurse completely disappeared under the priority restriction scenario, when turnout was about $8.7 \%$ lower. Likewise, the consequence of losing one nurse-126.1 forgone vaccinations in the baseline-was 77.7 vaccinations less severe under the priority restriction scenario. These results indicate that changes in patient demand have a very strong effect on the MPL, and that the marginal impact of a unit change in staffing is not symmetric with respect to the direction of change.

The results in the surge scenario show a similar interaction between nursing levels and patient demand. The $15 \%$ surge in demand alone led to an extra 69 patients being treated. The increase in treated patients likely results from the model's assumption that staff work slightly faster in response to seeing longer lines. If this assumption is incorrect, a surge might not lead to any additional patients being treated compared with a clinic that is already close to full capacity. The surge also amplified the effect of changes in nursing levels: Adding a nurse led to 45.4 more vaccinations than would have been added by an extra nurse in the baseline scenario, and removing a nurse led to 35.8 more vaccinations lost than the 126.1 lost under regular demand. Although average hourly clerk and nurse productivity was close to 190 and 150 patients served, respectively, in no scenarios is the effect of changing clerk levels close to the average clerk productivity, and only in some scenarios is the effect of adjusting nurse levels comparable.

The vaccination count declined by 18.44 when the percentage of patients who are contagious was incremented; this drop reflects the effect of not counting vaccinations given to the additional people who are infected or who become infected. Extending operating hours by $60 \mathrm{~min}$, which is almost a $10 \%$ increase in the amount of time the facility's doors are open, led to a $6.3 \%$ increase in vaccinations.

5.3.2. Average time in system. The decline in patient turnout under the priority restriction scenario led patients' ATS to fall by $37.7 \mathrm{~min}$, or $41.5 \%$. In contrast, the $15 \%$ surge in patient demand only increased ATS by $18.68 \mathrm{~min}$, or $20.6 \%$. Extending operating hours by $1 \mathrm{~h}$ further reduced average wait times by $7.48 \mathrm{~min}$, or $8.2 \%$. Removing and adding one nurse had a symmetrical effect of roughly $15 \mathrm{~min}$ in either direction. Removing a clerk increased ATS by $3.88 \mathrm{~min}$, while adding a clerk brought no benefit.

Under the priority restriction scenario, the benefit of adding a nurse was 5 min lower, and the effect of removing a clerk was 8.0 min stronger. The interaction between removing a clerk and the priority restriction sceario may be due to the shift in family sizes towards singles and couples, whose higher registration times could have increased the sensitivity of ATS to changes in clerk staffing levels. The coefficient for the interaction between removing a nurse and clerk simultaneously is negative, suggesting that while removing nurses and clerks one at a time increases ATS, the combined effect of removing both is less than the sum of the effects of removing them individually.

\subsubsection{Expected number of infections and infection risk.}

Several factors affected the expected number of infections, in part by reducing the number of patients arriving at the MIC_not necessarily a good thing_-but also by reducing 
Table 9 Factors affecting number vaccinated, ATS, expected infections, direct costs per vaccination, and total societal costs per vaccination

\begin{tabular}{|c|c|c|}
\hline Significant factors & Change from baseline & p-value \\
\hline \multicolumn{3}{|l|}{ Number vaccinated } \\
\hline Turnout representative of population & 31.48 & $<0.001$ \\
\hline Restriction to priority groups & -144.55 & $<0.001$ \\
\hline Increment percent infectious & -18.44 & $<0.001$ \\
\hline No patients infectious & 31.85 & $<0.001$ \\
\hline Extra hour & 105.05 & $<0.001$ \\
\hline Remove one nurse & -126.06 & $<0.001$ \\
\hline Add one nurse & 50.737 & $<0.001$ \\
\hline Remove one clerk & -39.25 & 0.0045 \\
\hline $15 \%$ surge in patient demand & 69.28 & $<0.001$ \\
\hline Interaction: priority restriction AND remove one nurse & 77.75 & $<0.001$ \\
\hline Interaction: priority restriction AND add one nurse & -68.31 & $<0.001$ \\
\hline Interaction: representative turnout AND add one nurse & 29.53 & 0.025 \\
\hline Interaction: add one nurse AND add one clerk & 28.25 & 0.032 \\
\hline Interaction: surge AND remove one nurse & -35.76 & 0.0011 \\
\hline Interaction: surge AND add one nurse & 45.44 & $<0.001$ \\
\hline \multicolumn{3}{|l|}{ Average time in system } \\
\hline Turnout representative of population & 5.18 & 0.0021 \\
\hline Restriction to priority groups & -37.7 & $<0.001$ \\
\hline Extra hour & -7.48 & $<0.001$ \\
\hline Remove one nurse & 14.97 & $<0.001$ \\
\hline Add one nurse & -14.86 & $<0.001$ \\
\hline Remove one clerk & 3.88 & 0.021 \\
\hline $15 \%$ surge in patient demand & 18.68 & $<0.001$ \\
\hline Interaction: priority restriction AND add one nurse & 4.99 & 0.012 \\
\hline Interaction: priority restriction AND remove one clerk & 8.01 & $<0.001$ \\
\hline Interaction: remove one nurse AND remove one clerk & -4.96 & 0.013 \\
\hline \multicolumn{3}{|l|}{ Expected infections } \\
\hline Restriction to priority groups & -5.08 & $<0.001$ \\
\hline Increment percent infectious & 3.56 & $<0.001$ \\
\hline No patients infectious & No infections & automatic \\
\hline Add one nurse & -1.05 & 0.013 \\
\hline $15 \%$ surge in patient demand & 2.53 & $<0.001$ \\
\hline Interaction: priority restriction AND increment $\%$ infectious & -1.12 & 0.040 \\
\hline Interaction: priority restriction AND remove one nurse & 1.27 & 0.020 \\
\hline Interaction: surge AND increment percent infectious & 0.97 & 0.032 \\
\hline \multicolumn{3}{|l|}{ Direct cost per vaccination } \\
\hline Increment percent infectious & 0.592 & $<0.001$ \\
\hline No patients infectious & -1.23 & $<0.001$ \\
\hline Add one clerk & 0.156 & 0.0029 \\
\hline Interaction: priority restriction AND none infectious & 0.372 & $<0.001$ \\
\hline Interaction: priority restriction AND add one nurse & 0.382 & $<0.001$ \\
\hline Interaction: surge AND none infectious & -0.162 & 0.031 \\
\hline Interaction: surge AND add one nurse & -0.223 & 0.0030 \\
\hline Interaction: surge AND remove one clerk & -0.206 & 0.0063 \\
\hline \multicolumn{3}{|l|}{ Total societal cost per vaccination } \\
\hline Turnout representative of population & 0.67 & 0.027 \\
\hline Restriction to priority groups & -6.35 & $<0.001$ \\
\hline Increment percent infectious & 0.58 & $<0.001$ \\
\hline No patients infectious & -1.62 & $<0.001$ \\
\hline Remove one nurse & 2.81 & $<0.001$ \\
\hline Add one nurse & -2.21 & $<0.001$ \\
\hline Remove one clerk & 0.61 & 0.046 \\
\hline $15 \%$ surge in patient demand & 3.39 & $<0.001$ \\
\hline Extra hour & -1.36 & $<0.001$ \\
\hline Interaction: priority restriction AND add one nurse & 1.24 & $<0.001$ \\
\hline Interaction: priority restriction AND remove one clerk & 1.43 & $<0.001$ \\
\hline Interaction: remove one nurse AND remove one clerk & -0.85 & 0.020 \\
\hline Interaction: surge AND add one nurse & -0.70 & 0.020 \\
\hline
\end{tabular}


patients' ATS. The baseline expected number of infections was 9.08 , representing $0.55 \%$ of those vaccinated. The priority restriction policy cut expected infections by $55.9 \%$, and resulted in a relative decline in infection risk per patient of $51.7 \%$. Incrementing the percentage of patients infected from 2 to $3 \%$ led to 3.56 new infections on average, an increase of $39.2 \%$. The $15 \%$ surge in patient demand led to a $27.9 \%$ increase in infections and a $22.7 \%$ increase in per patient infection risk, due largely to longer ATS. Adding one nurse averted 1.05 infections, which seems small but is an $11.6 \%$ improvement. Furthermore, from a societal perspective, the extra infected person's lost productivity over several days is likely of greater economic value than the cost of the additional nurse.

Several factor interactions were also present. The effects of the surge and of incrementing the percentage of infectious patients amplified each other slightly. The consequence of increasing the percentage of infectious patients was less severe in the priority restriction scenario. The reduction in expected infections from 9.08 to 4.00 under the priority restriction scenario was 1.27 infections less when one nurse was removed, though the effect of removing a nurse was not statistically significant in the baseline scenario.

\subsubsection{Direct and societal costs per vaccination. Although} four factors had statistically significant effects on the cost per vaccination, the effects were fairly modest. The cost per vaccination is $7.4 \%$ higher in the baseline scenario than when no infectious patients are in the model, a fact which indicates that the small percentage of vaccines given to people who will not benefit from them has economically meaningful implications. Adding a clerk to the baseline scenario increased the cost per vaccination, as did adding one nurse in the priority restriction scenario since demand was lower. Meanwhile, the cost per vaccination decreased in the surge scenario when a nurse was added and when a clerk was removed, indicating that the MIC may have best coped with a surge, given limited resources, by exchanging a clerk for a nurse, bringing the nurse:clerk ratio to $3: 2$.

If the economic loss due to patients waiting is taken into consideration, adding a clerk no longer hurts the cost per vaccination, and the benefit of adding a nurse is larger: $6.8 \%$ improvement in the baseline cost level and a $9.0 \%$ improvement in the surge cost level as opposed to no improvement in the baseline and a $1 \%$ improvement in the surge scenario. The factors that affected ATS also affected the societal cost per vaccine, and in similar ways. This cost per vaccine measure strikes a balance between higher patient throughput per worker per hour and keeping wait times short.

\section{Conclusions}

The simulation experiment's results show that making MIC staffing decisions on the basis of reaching vaccination targets, without considering the societal/economic losses associated with long waits or the possibility of intra-facility infection risk, could lead to under-investment in MICs. If an hour of a patient's time is valued at the minimum wage, then the cost of wait times of the length seen during the busiest days of the 2009 $\mathrm{H} 1 \mathrm{~N} 1$ pandemic becomes comparable with the direct financial cost of MIC staff, space rental, and vaccine materials combined. In addition to reducing wait-times, adding staff in the right circumstances also modestly reduced the expected number of intra-facility infections.

The analysis of staff productivity data revealed that there could be potential to increase patient throughput at MICs without hiring additional staff. Even though staff appeared to be working at full speed throughout the day, staff members' productivity varied considerably throughout the day, across different days, and between individuals. However, the reasons for these productivity variations remain unclear.

This simulation study is the first to estimate the risk of influenza transmission in a mass immunization clinic. As with all simulation studies, the results presented are contingent on the model's parameters and simplifying assumptions. Significant potential exists for gains in model accuracy and precision on several fronts. Transmission hazard rates within a 2-m radius are likely not well represented because virus-laden droplet and/ or aerosol density in the air will become more diffuse as the planar surface area, or volume, of space surrounding an infectious person increases with distance. The possibility of different infection risks for persons standing in front of an infected person versus behind was also not considered.

Possible improvements to the simulation model include the incorporation of direction-specific disease transmission (ie, disease transmission is more likely to occur in face-to-face orientations rather than facing the same direction in a queue), increased accuracy in the estimates of outdoor waiting times, and empirical verification of the relationship between perceived future workload, as represented by queue length, and hourly staff productivity. Data-based reneging and balking parameter estimates could also improve the bounds on queue growth, and the accuracy of the public's estimated time loss. That patients are recorded only after waiting outside is a major obstacle to assessing reneging behaviour. The use of appointment-based vaccination scheduling in conjunction with walk-ins could also be examined. An appointment plus walk-in approach might reduce variability in patient turnout, reduce wait times, and also generate reneging data.

Although the validity of the infection risk estimates produced by this simulation is subject to uncertainty, the estimates remain useful for comparing relative infection risk changes under different scenarios, and offer a reasonable approximation for actual infection counts using the best influenza transmission risk parameters available. It was found that no more than $1 \%$ of MIC patients are likely to become infected with influenza, even in a worst-case scenario in which $3 \%$ of other patients are infectious and without symptoms in addition to very long wait times. The risk of infection at an MIC does not outweigh the 
benefits of vaccination, even in a worst-case scenario. It is difficult to conceive of a plausible situation that would make the MICs counter-productive to pandemic control efforts. However, the risk of infection is still large enough to deserve attention from infection control experts, and is one of many factors to consider in hiring additional staff to reduce wait times. By ensuring adequate spacing between patients in waiting areas, good ventilation, sanitation of touch surfaces, etc, MIC managers may be able to increase the net positive effect of their facilities on pandemic control efforts by reducing intra-facility infection risk.

\section{References}

Aleman DM, Wibisono TG and Schwartz B (2011). A nonhomogeneous agent-based simulation approach to modeling the spread of disease in a pandemic outbreak. Interfaces 41(3): 301-315.

Amico C (2009). How much will the H1N1 flu cost the U.S.? PBS News, 8 October http://www.pbs.org/newshour/updates/health/july-dec09/ flu-costs_10-08.html, accessed 5 February 2012.

Andress K (2003). A postevent smallpox mass vaccination clinic exercise. Disaster Management Response 1(2): 54-58.

Araz OM, Lant T, Fowler JW and Jehn M (2011). A simulation model for policy decision analysis: A case of pandemic influenza on a university campus. Journal of Simulation 5(2): 89-100.

Asaro PV, Lewis LM and Boxerman SB (2008). Emergency department overcrowding: Analysis of the factors of renege rate. Academic Emergency Medicine 14(2): 157-162.

Asllani A, Dileepan P and Ettkin L (2007). A methodology for using simulation to optimize emergency mass vaccination parameters. Journal of Medical Systems 31(6): 453-459.

Barnes S, Golden B and Wasil E (2010). A dynamic patient network model of hospital-acquired infections. In: Johansson B, Jain S and Montoya-Torres J (eds). Proceedings of the 2010 Winter Simulation Conference (WSC 2010). Winter Simulation Conference, Baltimore, MD, pp 2249-2260.

Bearden A et al (2012). An outbreak of the 2009 influenza A (H1N1) virus in a children's hospital. Influenza and Other Respiratory Viruses 6(5): 374-379.

Blackwell T (2010). Ottawa H1N1 spending was too high, ineffective: critics. National Post, 6 April. http://www.nationalpost.com/news/ story.html?id=2770570, accessed 5 February 2012.

Brankston G, Gitterman L, Hirji Z, Lemieux C and Gardam M (2007). Transmission of influenza $\mathrm{A}$ in human beings. Lancet Infectious Disease 7(4): 257-265.

CBC News (2009). Canada to order 50.4 million H1N1 vaccine doses; $\$ 400 \mathrm{~m}$ contract goes to GlaxoSmithKline factory in Quebec City. http://www.cbc.ca/news/health/story/2009/08/06/swine-flu-vaccine .html, accessed 3 January 2012.

Centers for Disease Control and Prevention (2010). Interim guidance on infection control measures for $2009 \mathrm{H} 1 \mathrm{~N} 1$ influenza in healthcare settings, including protection of healthcare personnel. http://www.cdc .gov/h1n1flu/guidelines_infection_control.htm, accessed 8 November 2012.

Centers for Disease Control and Prevention (2011). CDC guidelines for large-scale influenza vaccination clinic planning. http://www.cdc.gov/ flu/professionals/vaccination/vax_clinic.htm, accessed 6 November 2012.

Cui F et al (2011). Transmission of pandemic influenza A (H1N1) virus in a train in China. Journal of Epidemiology 21(4): 271-277.

Das TK, Savachkin AA and Zhu Y (2008). A large scale simulation model of pandemic influenza outbreaks for development of dynamic mitigation strategies. IIE Transactions 40(9): 893-905.
Durbin A, Corallo A, Wibisono T, Aleman D, Schwartz B and Coyte P (2011). A cost effectiveness analysis of the H1N1 vaccine strategy for Ontario, Canada. Journal of Infectious Diseases and Immunity 3(3): 40-49.

Elveback LR, Fox JP, Ackerman E, Langworthy A, Boyd M and Gatewood L (1976). An influenza simulation model for immunization studies. American Journal of Epidemiology 103(2): 152-165.

Fomundam S and Herrmann JW (2007). A survey of queuing theory applications in healthcare. Technical Report TR 2007-24, Institute for Systems Research, University of Maryland. URL http://drum.lib .umd.edu/handle/1903/7222, accessed 28 August 2013.

Foxwell RA, Roberts L, Lokuge K and Kelly PM (2011). Transmission of influenza on international flights, May 2009. Clinical Infectious Diseases 17(7): 1188

Gentry A (2010). PECS data monitoring system. Internal Document, Niagara Region Public Health.

Gentry A (2011). Protocol for electronic clinic systems version 3.0 - data analysis. Internal Document, Niagara Region Public Health.

Gojovic MZ, Sander B, Fisman D, Krahn MD and Bauch CT (2009). Modelling mitigation strategies for pandemic (H1N1) 2009. Canadian Medical Association Journal 181(10): 673-680.

Haber MJ et al (2007). Effectiveness of interventions to reduce contact rates during a simulated influenza pandemic. Emerging Infectious Disease 13(4): 581-589.

Hupert N, Mushlin A and Callahan M (2002). Modeling the public health response to bioterrorism: using discrete event simulation to design antibiotic distribution centers. Medical Decision Making 22(5): s17-25.

Influenza Team, European Centre for Disease Prevention and Control (2007). Influenza transmission: re-search needs for informing infection control policies and practice. Eurosurveillance 12: 3189.

Khan K et al (2011). An analysis of canada's vulnerability to emerging infectious disease threats via the global airline transportation network. Scientific report, St. Michael's Hospital.

Klontz KC, Hynes NA, Gunn RA, Wilder MH, Harmon MW and Kendal AP (1989). An outbreak of influenza A/Tiawan/1/86 (H1N1) infections at a naval base and its association with airplane travel. American Journal of Epidemiology 129(2): 341-349.

Knibbs D, Morawska L and Bell S (2012). The risk of airborne influenza transmission in passenger cars. Epidemiology and Infection 140(3): 474-475.

Las Vegas Review-Journal (2009). Long lines mark first day of H1N1 vaccine shots. http://www.lvrj.com/news/breaking_news/Long-linesmark-first-day-of-H1N1-vaccine-shots-64673797.html, accessed 7 November 2012.

Lee B Y et al (2010). A computer simulation of vaccine prioritization, allocation, and rationing during the $2009 \mathrm{H} 1 \mathrm{~N} 1$ influenza pandemic. Vaccine 28(31): 4875-4879.

Lessler J, Reich NG and Cummings DA the New York City Department of Health and Mental Hygiene Swine Influenza Investigation Team (2009). Outbreak of 2009 pandemic influenza A (H1N1) at a New York City school. The New England Journal of Medicine 361(27): 2628-2636.

Los Alamos National Laboratory (2006). EpiSimS Los Angeles case study. http://public.lanl.gov/stroud/LACaseStudy5.pdf, accessed 15 March 2011.

Mei S, Van De Vijver D, Xuan L, Zhu Y and Sloot PMA (2010). Quantitatively evaluating interventions in the influenza A (H1N1) epidemic on China campus grounded on individual-based simulations. In: Sloot PMA, van A Albada GD and Dongarra J (eds). Proceedings of the International Conference on Computational Science (ICCS 2010). International Conference on Computational Science, Amsterdam, pp 1675-1682.

Mitchell R et al (2012). Impact of the 2009 influenza A (H1N1) pandemic on Canadian health care workers: A survey on vaccination, illness, absenteeism, and personal protective equipment. American Journal of Infection Control 40(7): 611-616. 
Moser MR, Bender TR, Margolis HS, Noble GR, Kendal AP and Ritter DG (1979). An outbreak of influenza aboard a commercial airliner. American Journal of Epidemiology 110(1): 1-6.

Mylius S, Hagenaars T, Lugner A and Wallinga J (2008). Optimal allocation of pandemic influenza vaccine depends on age, risk, and timing. Vaccine 26(30): 3742-3749.

Noakes CJ and Sleigh PA (2009). Mathematical models for assessing the role of airflow on the risk of airborne infection in hospital wards. Journal of the Royal Society, Interface 6(S6): S791-S800.

NY1 News (2009). Long lines wait for H1N1 vaccines at weekend clinics. http://queens.ny1.com/content/top_stories/108933/long-lineswait-for-h1n1-vaccines-at-weekend-clinics, accessed 7 November 2012

Phillips FB and Williamson JP (2005). Local health department applies incident management system for successful mass influenza clinics. Journal of Public Health Management Practice 11(4): 269-273.

Public Health Agency of Canada (2010). Lessons learned review: Public Health Agency of Canada and Health Canada Response to the 2009 H1N1 pandemic. http://www.phac-aspc.gc.ca/about_apropos/ evaluation/reports-rapports/2010-2011/h1n1/context-contexte-eng.php \#figure-2-2, accessed 11 November 2012.

Public Health Agency of Canada (2011). The Canadian pandemic influenza plan for the health sector. http://www.phac-aspc.gc.ca/cpippclcpi/index-eng.php, accessed 6 November 2012.

Salgado CD, Farr BM, Hall KK and Hayden FG (2002). Influenza in the acute hospital setting. The Lancet Infectious Diseases 2(3): 145-155.

Sander B et al (2010). Is a mass immunization program for pandemic (H1N1) 2009 good value for money? Evidence from the Canadian experience. Vaccine 28(38): 6210-6220.

Savachkin A and Uribe-Sanchez Andres (2012). Dynamic redistribution of mitigation resources during influenza pandemics. Socio-economic Planning Sciences 46(1): 33-45.

Sikora C et al (2010). Transmission of pandemic influenza A (H1N1) 2009 within households: Edmonton, Canada. Journal of Clinical Virology 49(2): 90-93.

Statistics Canada (2006). Census 2006. http://www12.statcan.ca/censusrecensement/2006/rt-td/ index-eng.cfm, accessed 15 March 2011.
Stroud P, Del Valle S, Sydoriak S, Riese J and Mniszewski S (2007). Spatial dynamics of pandemic influenza in a massive artificial society. Journal of Artificial Societies and Social Simulation 10(4): 9.

Tellier R (2006). A review of aerosol transmission of influenza A virus. Emerging Infectious Diseases 12(11): 1657-1662.

United Kingdom Department of Health (2011). UK Influenza Pandemic Preparedness Strategy 2011. http://www.dh.gov.uk/prod_consum_dh/ groups/dh_digitalassets/documents/digitalasset/dh_131040.pdf, accessed 6 November 2012.

Viboud C et al (2004). Risk factors of influenza transmission in households. The British Journal of General Practice 54(506): 684-689.

Waldie P and Alphonso C (2009). Cost of vaccinating nation hits $\$ 1.5$ billion and climbing. Globe and Mail, 12 November 2009. http://www.theglobeandmail.com/life/health-and-fitness/health/ conditions/cost-of-vaccinating-the-nation-hits-15-billion-and-climbing/ article1318824/, accessed 5 February 2012.

Washington M, Mason J and Meltzer M (2005). Maxi-Vac: planning mass smallpox vaccination clinics. Journal of Public Health Management Practice 11(6): 542-549.

Washington ML (2009). Evaluating the capability and cost of a mass influenza and pneumococcal vaccination clinic via computer simulation. Medical Decision Making 29: 414-423.

Wong BC et al (2010). Possible role of aerosol transmission in a hospital outbreak of influenza. Clinical Infectious Diseases 51: 1176-1183.

World Health Organization (2012). Infection prevention and control in health care. http://www.who.int/csr/bioriskreduction/infection_control/ en/index.html, accessed 8 November 2012.

Yang J (2009). First-day of H1N1 shots meant 3 hours of waiting. http:// www.healthzone.ca/health/newsfeatures/swineflu/article/716547, accessed 7 November 2012.

Zenios SA (1999). Modeling the transplant waiting list: A queueing model with reneging. Queuing Systems 31(3): 239-251.

Received 10 January 2012; accepted 1 October 2013 after two revisions 